\title{
Environmental risk factors for Toxoplasma gondii infections and the impact of latent infections on allostatic load in residents of Central North Carolina
}

Andrey I. Egorov ${ }^{1 *} \mathbb{D}$, Reagan Converse ${ }^{1}$, Shannon M. Griffin ${ }^{2}$, Jennifer Styles ${ }^{1,3}$, Elizabeth Klein ${ }^{4}$, Elizabeth Sams ${ }^{1}$, Edward Hudgens ${ }^{1}$ and Timothy J. Wade ${ }^{1}$

\begin{abstract}
Background: Toxoplasma gondii infection can be acquired through ingestion of infectious tissue cysts in undercooked meat or environmental oocysts excreted by cats. This cross-sectional study assessed environmental risk factors for $T$. gondii infections and an association between latent infections and a measure of physiologic dysregulation known as allostatic load.

Methods: Serum samples from 206 adults in the Durham-Chapel Hill, North Carolina area were tested for immunoglobulin (lgG) responses to T. gondii using commercial ELISA kits. Allostatic load was estimated as a sum of 15 serum biomarkers of metabolic, neuroendocrine and immune functions dichotomized at distribution-based cutoffs. Vegetated land cover within $500 \mathrm{~m}$ of residences was estimated using $1 \mathrm{~m}$ resolution data from US EPA's EnviroAtlas.

Results: Handling soil with bare hands at least weekly and currently owning a cat were associated with 5.3 (95\% confidence limits $1.4 ; 20.7)$ and $10.0(2.0 ; 50.6)$ adjusted odds ratios (aOR) of T. gondii seropositivity, respectively. There was also a significant positive interaction effect of handling soil and owning cats on seropositivity. An interquartile range increase in weighted mean vegetated land cover within $500 \mathrm{~m}$ of residence was associated with $3.7(1.5 ; 9.1)$ aOR of T. gondii seropositivity. Greater age and consumption of undercooked pork were other significant predictors of seropositivity. In turn, T. gondii seropositivity was associated with 61\% (13\%; 130\%) greater adjusted mean allostatic load compared to seronegative individuals. In contrast, greater vegetated land cover around residence was associated with significantly reduced allostatic load in both seronegative $(p<0.0001)$ and seropositive $(p=0.004)$ individuals.

Conclusions: Residents of greener areas may be at a higher risk of acquiring T. gondii infections through inadvertent ingestion of soil contaminated with cat feces. T. gondii infections may partially offset health benefits of exposure to the natural living environment.
\end{abstract}

Keywords: Toxoplasma gondii, Seroprevalence, Vegetated land cover, Biomarkers, Allostatic load

\footnotetext{
* Correspondence: egorov.andrey@epa.gov

${ }^{1}$ United States Environmental Protection Agency, National Health and Environmental Effects Research Laboratory, MD 58-C, 109. T.W. Alexander Drive, Research Triangle Park, NC 27711, USA

Full list of author information is available at the end of the article
}

(c) The Author(s). 2018 Open Access This article is distributed under the terms of the Creative Commons Attribution 4.0 International License (http://creativecommons.org/licenses/by/4.0/), which permits unrestricted use, distribution, and reproduction in any medium, provided you give appropriate credit to the original author(s) and the source, provide a link to the Creative Commons license, and indicate if changes were made. The Creative Commons Public Domain Dedication waiver (http://creativecommons.org/publicdomain/zero/1.0/) applies to the data made available in this article, unless otherwise stated. 


\section{Background}

Toxoplasma gondii is a ubiquitous protozoan parasite that infects felines as definitive hosts and a wide range of other warm-blooded animals, including humans, as intermediate hosts. Infected cats excrete copious amounts of T. gondii oocysts containing infectious sporozoites in their feces. The oocysts can contaminate soils and remain viable for months to years $[1,2]$; subsequent ingestion of oocyst-contaminated soil causes infection in intermediate hosts. T. gondii infects many tissues of intermediate hosts, including muscles and the central nervous system, where it forms infectious tissue cysts. Predation of intermediate hosts by felines completes the life cycle of the parasite. In humans, life-long infections usually result from ingestion of raw or undercooked meat of infected intermediate hosts, such as pigs, as well as ingestion of environmental oocysts [3]. Other infection routes in humans include vertical transmission from infected mother to her fetus and transmission via blood transfusion or organ transplant $[4,5]$. A new infection typically involves a transient acute phase caused by the rapidly replicating tachyzoites of the parasite followed by a latent, life-long stage with bradyzoites persisting inside tissue cysts.

Serum immunoglobulin (Ig) $M$ response to the parasite is characteristic of the acute phase of infection. Serum IgG response, which reaches a maximum level within 2 to 3 months of initial infection and then slowly declines to a residual elevated level, is characteristic of the latent phase of infection [4]. A combination of serum IgG and IgM responses to the parasite is used to differentiate acute and latent infection phases in diagnostic settings [6]. Serum IgG immunoassays are a standard test used in population surveillance of $T$. gondii seroprevalence [7, 8].

According to the nationally representative data from the National Health and Nutrition Examination Survey (NHANES), IgG seroprevalence of T. gondii in the US in individuals older than 5 years of age was $13.2 \%$ in 20092010. There had been a substantial decline in seroprevalence during the previous 20-year interval [8]. Low socioeconomic status has been linked with increased odds of seropositivity in the US [9].

There is strong epidemiologic evidence of $T$. gondii transmission via consumption of raw or undercooked meat $[10,11]$ suggesting that ingestion of tissue cysts is a dominant infection pathway in the US and other developed countries. Research using antibody responses to sporozoite-specific antigen demonstrated that ingestion of environmental oocysts containing sporozoites is also a common infection pathway in North America [12, 13]. Some studies also provided evidence of an association between contacts with cats and $T$. gondii infection in developed countries [10,11, 14], while other studies failed to confirm this association [15-17]. Associations between gardening and other soil contacts with risk of $T$. gondii infection have also been demonstrated [15, 16, 18]. In addition, waterborne outbreaks of toxoplasmosis have been reported in Canada [19] and Brazil [20].

Only $10-30 \%$ of new T. gondii infections in humans cause clinical symptoms [21], but when symptoms are present, the clinical manifestations of the disease can be severe. Symptoms of acute toxoplasmosis include ocular disease, encephalitis, chorioretinitis, lymphadenitis or lymphadenopathy, and myocarditis [22]. T. gondii infection during pregnancy and vertical transmission of the parasite to the fetus can cause mental disabilities, seizures, blindness and spontaneous abortion [3]. In the US, 400 to 4000 infants are born annually with congenital toxoplasmosis [3, 23].

While the latent phase of infection may appear asymptomatic, there is a growing body of evidence of behavioral modifications in intermediate hosts enhancing the probability of predation by felines. Examples from animal studies include fatal attraction to and sexual arousal by the smell of cat urine in infected rats [24, 25], and similar fatal attraction to the smell of leopard urine in infected chimpanzees [26]. Epidemiological studies have also linked latent infections in humans with adverse neuropsychological outcomes, including elevated risk of suicide [27], impaired reaction time and increased risk of traffic accidents [28], and mental health disorders including schizophrenia, depression, and obsessive compulsive disorder [22], as well as greater odds of developing a metabolic disorder type 2 diabetes [29]. There is also evidence of widespread immune activation and subclinical neurophysiological changes induced by $T$. gondii infection in humans [30, 31]. However, the knowledge of sub-clinical health effects of latent infections and biological pathways leading to these effects remains rather limited.

The objectives of this study were: (i) to assess behavioral and environmental risk factors for $T$. gondii infections in the Durham-Chapel Hill, NC area, and (ii) to explore potential associations between latent $T$. gondii infections and a composite biomarker-based measure of physiological dysregulation known as allostatic load (AL), and individual biomarkers of immune, neuroendocrine and metabolic functions.

\section{Methods}

\section{Study design and data collection}

The protocol of this cross-sectional population-based observational study was approved by the Institutional Review Board of the University of North Carolina at Chapel Hill. The target population included adult (at least 18 years of age) residents of the Durham-Chapel Hill metropolitan area in North Carolina. The study was advertised on the US EPA's website for recruitment of volunteers in epidemiological research, and by displaying study posters at various venues. Veterinary practices and 
animal shelters were targeted for recruitment to over-sample individuals with increased contacts with cats, dogs and other animals. Participants reported to the US EPA Human Studies Facility (HSF) in Chapel Hill, NC. A venous blood sample was drawn in a BD Vacutainer SST tube (Becton, Dickinson and Company, Franklin Lakes, NJ), and height and weight were measured by a registered nurse. Serum was separated following manufacturer instructions on the day of collection, and stored at $-80^{\circ} \mathrm{C}$ until analysis. Participants also completed a questionnaire addressing their demographic and socioeconomic characteristics, and behavioral and environmental factors which may be associated with exposure to $T$. gondii, such as contacts with cats, handling soil and consumption of undercooked meat of various types. Data collection was conducted in May - September 2013.

\section{Serological tests}

Serum samples were tested for IgG response to $T$. gondii using VIR-ELISA Anti-Toxo IgG assays (VIRO-IMMUN Labor-Diagnostika GmbH, Oberursel, Germany) in accordance with manufacturer's instructions. Geometric mean values from duplicate tests were used in data analysis. Samples from two individuals with indeterminate results (average optical density values within plus/minus $10 \%$ interval around the plate-specific cut-off) were re-analyzed. If a new test again produced an indeterminate result, the infection status was classified as negative if the average ratio of optical density value for the sample to the corresponding plate-specific cut-off was less than one and as positive otherwise.

As part of a previously conducted study of environmental predictors of AL [32], serum samples were also analyzed for 15 stress-related biomarkers, including nine biomarkers of immune function: C-reactive protein (CRP), vascular cell adhesion molecule 1 (VCAM-1), intercellular adhesion molecule 1 (ICAM-1), interleukin (IL)-1 $\beta$, IL-6, IL-8, tumor necrosis factor (TNF)- $\alpha$, fibrinogen, and myeloperoxidase (MPO); four biomarkers of neuroendocrine function: dehydroepiandrosterone (DHEA), epinephrine, norepinephrine, and dopamine; and two biomarkers of metabolic function: uric acid and serum amyloid A (SAA). All biomarker tests were conducted using commercially available assay kits as described previously [32].

\section{Analysis of allostatic load}

AL was calculated as a sum of dichotomized biomarker values, which is the most commonly used approach in $\mathrm{AL}$ studies [33]. Biomarker data were dichotomized at the 10th percentile of the sample distribution (DHEA and dopamine), at the 90th percentile (IL-1 $\beta$, IL-6, IL-8, TNF- $\alpha$, fibrinogen, uric acid, MPO, CRP, SAA, VCAM-1, and ICAM-1) or at both 10th and 90th percentiles (norepinephrine and epinephrine, two binary variables for each biomarker), depending on which tail of the biomarker distribution is known to be associated with an elevated risk of disease or death. Thus, AL measures were based on a total of 17 binary variables representing 15 biomarkers as described previously [32].

\section{Analysis of residential exposure to living environment}

Proportions of total vegetated land cover within a $500 \mathrm{~m}$ radius of each residence were estimated using high resolution land cover data for the Durham-Chapel Hill, NC metropolitan area from the US EPA's mapping application EnviroAtlas (https://www.epa.gov/enviroatlas) as described previously [32]. Vegetated land cover was defined as the sum of two land cover categories: Trees \& forest, and Grass \& other herbaceous. Exposure measures were based on average proportion of vegetated land cover within $50 \mathrm{~m}, 150 \mathrm{~m}$, and $500 \mathrm{~m}$ radii, and distance-weighted average proportion of vegetated land cover within $500 \mathrm{~m}$ radius around the residence. The latter measure was calculated as an arithmetic mean of vegetated land cover proportions for ten concentric $50 \mathrm{~m}$ annuli from $0-50 \mathrm{~m}$ to $450-500 \mathrm{~m}$. This weighting scheme implicitly used a constant weight of 0.1 for each annulus. As a result, a square meter of vegetated land cover within the $0-50 \mathrm{~m}$ annulus $\left(7854 \mathrm{~m}^{2}\right.$ area) had 19 times greater impact on the weighted estimate than a square meter of vegetated land cover within the 450$500 \mathrm{~m}$ annulus with 19 times larger area $\left(149,226 \mathrm{~m}^{2}\right)$.

\section{Statistical data analysis}

Statistical analysis was conducted using SAS version 9.4 software (SAS Institute, Cary, NC). It involved two phases: the first phase focused on environmental predictors of $T$. gondii infections with $T$. gondii serostatus being an outcome variable while the second phase focused on subclinical health outcomes of latent $T$. gondii infections. In the second phase, $T$. gondii serostatus was a predictor variable while AL and individual biomarkers were modeled as outcome variables, one variable at a time.

At the first phase, univariate analysis of associations between demographic, behavioral and environmental factors and T. gondii infections was conducted using the Chi-square Wald test for binary and nominal variables and the Cochran-Armitage test for trend for ordinal variables. Subsequent multivariate regression analysis involved developing predictive models of $T$. gondii seropositivity. An initial predictive logistic regression model included a set of socio-demographic and behavioral covariates. The next step involved adding cat-related variables to the initial model, one variable at a time, and selecting the cat variable that produced the best model fit. Akaike Information Criterion Corrected (AICc) values in the output of SAS procedure genmod were used as a measure of model fit. 
The next step involved adding vegetated land cover measures to the previously developed model, one variable at a time. To account for spatial autocorrelation, all regression models for vegetated land cover included a two-dimensional spline smoothing function of geographic coordinates ("thin-plate smoother"), as described previously [32]. The models involving a combination of linear and non-linear predictors also known as generalized additive models were fitted using the SAS procedure gam. Using generalized additive models is a common approach in analysis of geographic distributions of health outcomes [3436]. The spline function was fitted using the option "method = GCV" (generalized cross-validation function) for automatically selecting degrees of freedom which define the flexibility of the "thin plate" smoother. Model fit was assessed using the deviance of the final estimate criterion from the output of SAS procedure gam.

In the second phase, associations of T. gondii seropositivity with AL (a Poisson-distributed count variable), as well as individual dichotomized biomarkers and continuous log-transformed biomarkers were analyzed in univariate and in multivariate regression models adjusting for demographic and socioeconomic covariates. The univariate analysis of association between $T$. gondii and AL was followed by developing a multivariate predictive Poisson regression model including demographic covariates and body mass index (BMI). A final predictive model for AL also included vegetated land cover as a covariate and a spline function of geographic coordinates to account for spatial autocorrelation; it was fitted using the SAS procedure gam as described above. Associations between $T$. gondii seropositivity and individual biomarkers were analyzed using logistic regression models for binary biomarkers adjusting for socio-demographic covariates or linear multivariate models for log-transformed biomarker data.

\section{Results}

Predictors of $T$. gondii seropositivity Descriptive statistics, univariate analysis and associations among covariates

Two-hundred and six individuals residing at 173 street addresses were included in the present study. Among them, there were 17 (8.3\%) T. gondii seropositive individuals (Table 1). The mean age of participants was 37.4 years with a range from 18 to 85 years. As expected, $T$. gondii seropositivity was associated with increased age $(p<0.0001)$ in univariate analysis. Sex, race and ethnicity status (dichotomized as non-Hispanic Whites (53.7\%) vs. all others), and education (dichotomized as 4 -year college degree or greater $(51.5 \%)$ vs. less than college degree) were not associated with seropositivity. Obesity $(28.6 \%$ of participants) was significantly associated with seropositivity in a univariate model. Approximately half of the study participants (51.5\%) lived in Durham (city with 258,000 residents in 2015), 29.1\% lived in Chapel Hill (city with a large university campus; 59,000 residents), and the remaining $19.4 \%$ lived in other towns in the Durham-Chapel Hill metropolitan area. Seroprevalence rates did not differ significantly among these locations. Individuals who handled soil with bare hands at least one time per week were significantly more likely to be seropositive in the univariate analysis. While current cat ownership was a significant risk factor, past cat ownership was not associated with seropositivity. Other variables significantly associated with $T$. gondii in univariate analysis included having outdoor cats, and a total duration of cat ownership of 4 years or longer.

Stratified univariate analysis demonstrated that regularly handling soil with bare hands was associated with higher odds of $T$. gondii seropositivity only among current cat owners who allow their cats to go outdoors (Table 2). Among five T. gondii seropositive individuals who did not have outdoor cats and did not handle soil with bare hands regularly, none $(0 \%)$ were non-Hispanic White, four (80\%) were obese, and only one (20\%) attained at least bachelor's level education, while the average age in this group was 58.6 years. In contrast, among seven seropositive individuals who had outdoor cats and handled soil regularly, six (86\%) were non-Hispanic White, two (29\%) were obese, and four (57\%) attained a bachelor's level education, while the average age in this group was 39.7 years.

The median proportions of vegetated land cover within $50 \mathrm{~m}$ and $500 \mathrm{~m}$ around residence were 59.9 and $72.8 \%$, respectively (Table 3 ); statistics for $150 \mathrm{~m}$ radius and for the distance-weighted measure of land cover were between these estimates. Interquartile ranges (IQR) varied from $15.0 \%$ for average vegetated land cover within $500 \mathrm{~m}$ to $25.9 \%$ for the $50 \mathrm{~m}$ radius. Average housing unit density at a census block-group level varied from 0.08 to 11.2 (median 1.53, IQR 1.93) housing units per acre or from 0.20 to 27.7 (median 3.78) housing units per hectare.

Exploratory analysis of predictors of current cat ownership (dichotomized as at least one cat vs. no cats) demonstrated that current dog ownership (dichotomized similarly) was inversely associated with cat ownership ( $\mathrm{aOR}=0.10,95 \%$ confidence limits $0.04 ; 0.24$ ), while non-Hispanic White ethno-racial status was associated with a $16.1(7.00 ; 37.0)$ aOR of current cat ownership compared to non-White and Hispanic individuals. An IQR increase in housing unit density was linked with $0.53(0.29 ; 0.98)$ OR of owning a cat that roamed outdoors. Individuals with greater vegetated land cover around their residence were more likely to handle soil with bare hands at least weekly. The strongest effect was observed for distance-weighted proportion of vegetated 
Table 1 Descriptive statistics of the study population and univariate analysis of risk factors for T. gondii seropositivity

\begin{tabular}{|c|c|c|c|c|}
\hline Variable & Level & $\begin{array}{l}\text { Participants, } \\
\text { N (\%) }\end{array}$ & $\begin{array}{l}\text { T. gondii seropositive, } \\
\mathrm{N}(\%)\end{array}$ & $p$ value \\
\hline All participants & & $206(100 \%)$ & $17(8.3 \%)$ & \\
\hline \multirow[t]{3}{*}{ Age } & $18-30$ & $88(42.7 \%)$ & $2(2.3 \%)$ & \\
\hline & $31-45$ & $62(30.1 \%)$ & $5(8.1 \%)$ & \\
\hline & $46-85$ & $56(27.2 \%)$ & $10(17.9 \%)$ & $0.001^{\mathrm{a}}$ \\
\hline \multirow[t]{2}{*}{ Sex } & Males & $70(34.0 \%)$ & $5(7.1 \%)$ & \\
\hline & Females & $136(66.0 \%)$ & $12(8.8 \%)$ & 0.7 \\
\hline \multirow[t]{2}{*}{ Race and ethnicity } & White non-Hispanic & $110(53.4 \%)$ & $8(7.3 \%)$ & \\
\hline & All others & $95(46.1 \%)$ & $9(9.5 \%)$ & 0.6 \\
\hline \multirow[t]{2}{*}{ Education } & Less than bachelor's degree & $100(48.5 \%)$ & $10(10.0 \%)$ & \\
\hline & Bachelor's degree or higher & $106(51.5 \%)$ & $7(6.6 \%)$ & 0.4 \\
\hline \multirow[t]{2}{*}{ Obesity } & Not obese & $147(71.4 \%)$ & $8(5.4 \%)$ & \\
\hline & Obese & $59(28.6 \%)$ & $9(15.3 \%)$ & 0.03 \\
\hline \multirow[t]{2}{*}{ Smoker } & No & $148(71.8 \%)$ & $9(6.1 \%)$ & \\
\hline & Yes & $57(27.7 \%)$ & $8(14.0 \%)$ & 0.07 \\
\hline \multirow[t]{3}{*}{ Residence } & Chapel Hill & $60(29.1 \%)$ & $4(6.7 \%)$ & \\
\hline & Durham & $106(51.5 \%)$ & $10(9.4 \%)$ & \\
\hline & Other towns & $40(19.4 \%)$ & $3(7.5 \%)$ & 0.8 \\
\hline \multirow[t]{2}{*}{ Drinking water source at home } & Private well or bottled water & $67(32.5 \%)$ & $5(7.5 \%)$ & \\
\hline & Municipal system & $136(66.0 \%)$ & $12(8.8 \%)$ & 0.7 \\
\hline \multirow[t]{2}{*}{ Housing unit density } & $<4$ per acre & $178(86.4 \%)$ & $14(7.9 \%)$ & \\
\hline & $>=4$ per acre & $28(13.6 \%)$ & $3(10.7 \%)$ & 0.6 \\
\hline \multirow[t]{2}{*}{ Ever lived on a farm } & No & 169 (82.0\%) & $11(6.5 \%)$ & \\
\hline & Yes & $37(18.0 \%)$ & $6(16.2 \%)$ & 0.06 \\
\hline \multirow[t]{2}{*}{ Eat raw/undercooked chicken } & No & $187(90.8 \%)$ & $13(7.0 \%)$ & \\
\hline & Yes & $14(6.8 \%)$ & $3(21.4 \%)$ & 0.07 \\
\hline \multirow[t]{2}{*}{ Eat raw/undercooked pork } & No & $188(91.3 \%)$ & $14(7.4 \%)$ & \\
\hline & Yes & $12(5.8 \%)$ & $2(16.7 \%)$ & 0.27 \\
\hline \multirow[t]{2}{*}{ Eat raw/undercooked beef } & No & $127(61.7 \%)$ & $12(9.4 \%)$ & \\
\hline & Yes & $74(35.9 \%)$ & $4(5.4 \%)$ & 0.31 \\
\hline \multirow[t]{2}{*}{ Handles soil with bare hands weekly } & No & $151(73.3 \%)$ & $8(5.3 \%)$ & \\
\hline & Yes & $53(25.7 \%)$ & $8(15.1 \%)$ & 0.03 \\
\hline \multirow[t]{2}{*}{ Currently has at least one dog } & No & $62(30.1 \%)$ & $8(12.9 \%)$ & \\
\hline & Yes & $144(69.9 \%)$ & $9(6.3 \%)$ & 0.12 \\
\hline \multirow[t]{2}{*}{ Currently has at least one cat } & No & $115(55.8 \%)$ & $5(4.3 \%)$ & \\
\hline & Yes & $91(44.2 \%)$ & $12(13.2 \%)$ & 0.03 \\
\hline \multirow[t]{3}{*}{ Current outdoor cats } & Do not have cats & $115(55.8 \%)$ & $5(4.3 \%)$ & \\
\hline & Has cats that don't go outdoors & $30(14.6 \%)$ & $2(6.7 \%)$ & \\
\hline & Has cats, let them go outdoors & $61(29.6 \%)$ & $10(16.4 \%)$ & $0.007^{\mathrm{a}}$ \\
\hline \multirow[t]{2}{*}{ Total years lived with cats } & $0-3$ years & $115(55.8 \%)$ & $4(3.5 \%)$ & \\
\hline & 4 years or more & $91(44.2 \%)$ & $13(14.3 \%)$ & 0.01 \\
\hline \multirow[t]{2}{*}{ Ever been responsible for cleaning a cat litter box } & No & $75(36.4 \%)$ & $3(4.0 \%)$ & \\
\hline & Yes & $130(63.1 \%)$ & $13(10.0 \%)$ & 0.14 \\
\hline
\end{tabular}


Table 2 Stratified descriptive statistics on T. gondii seropositivity

\begin{tabular}{|c|c|c|c|c|}
\hline Strata & Substrata & Participants & T. gondii seropositive & $p$ value \\
\hline \multirow[t]{2}{*}{ No outdoor cats } & Does not handle soil weekly & $112(54.4 \%)$ & $5(4.5 \%)$ & \\
\hline & Handles soil with bare hands weekly & $31(15.0 \%)$ & $1(3.2 \%)$ & 0.76 \\
\hline \multirow[t]{2}{*}{ Outdoor cats present } & Does not handle soil weekly & 39 (18.9\%) & $3(7.7 \%)$ & \\
\hline & Handles soil with bare hands weekly & $22(10.7 \%)$ & $7(31.8 \%)$ & 0.02 \\
\hline
\end{tabular}

land cover within $500 \mathrm{~m}$ : an IQR increase in this measure of vegetated land cover was associated with a 1.68 $(1.04 ; 2.71)$ aOR of handling soil after adjusting for race/ ethnicity and spline function of geographic coordinates.

\section{Multivariate regression analysis of predictors of $T$. gondii seropositivity}

A preliminary predictive model of seropositivity included age, smoking status, obesity, eating undercooked pork, average housing unit density per census block group, and handling soil with bare hands at least weekly. Further analysis of cat-related variables identified currently owning a cat as the strongest predictor of $T$. gondii seropositivity. The resulting multivariate predictive model of seropositivity (Model 1) is presented in Table 4.

Further analysis involved assessing an interaction effect of handling soil with bare hands and being exposed to cats. In this analysis, a binary variable for owning a cat that roamed outdoors produced the best model fit (Model 2, Table 4). The interaction effect of handling soil and owning an outdoor cat on odds of seropositivity was statistically significantly positive $(p=0.01)$. The interaction effect of handling soil and owning any cats was also statistically significant with $p=0.03$ (not shown). Housing unit density was excluded from these models because preliminary analysis demonstrated that it was inversely associated with allowing cats to go outdoors.

Model 3 (Table 4) was developed to assess an association between vegetated land cover and T. gondii seropositivity. Preliminary analysis involved fitting four models with measures of average vegetated land cover within $50 \mathrm{~m}, 150 \mathrm{~m}$, or $500 \mathrm{~m}$ of residence, or $500 \mathrm{~m}$ distance-weighted average, one measure at a time (Table 5). The distance-weighted average measure produced the best model fit. In this model, an IQR increase ( $17.4 \%$ increase) in vegetated land cover was associated

Table 3 Percent of vegetated land cover within specified distance from residence

\begin{tabular}{llll}
\hline Measure of vegetated land cover & Median & Min - max & IQR \\
\hline 50 m average & 59.9 & $6.0-99.8$ & 25.9 \\
150 m average & 67.6 & $19.5-94.8$ & 21.7 \\
500 m average & 72.8 & $36.9-95.2$ & 15.0 \\
500 m weighted & 68.7 & $31.7-94.3$ & 17.4 \\
\hline
\end{tabular}

with a $3.67(1.48 ; 9.08)$ aOR of $T$. gondii seropositivity $(p=0.006)$. Obesity was excluded from Model 3 as it was no longer a significant predictor of seropositivity. Handling soil with bare hands was also excluded from Model 3 because preliminary analysis demonstrated that greater vegetated land cover was associated with greater odds of handling soil. An additional analysis demonstrated that adjusting for handling soil with bare hands reduced the effect estimate for vegetated land cover to $2.94(1.13 ; 7.68)$ indicating that more frequent contacts with soil contaminated with $T$. gondii oocysts partially mediated the observed association between residential vegetated land cover and $T$. gondii infections.

\section{Latent T. gondii infection as a predictor of physiologic dysregulation}

In this analysis, $T$. gondii serostatus was modelled as a predictor variable while outcome variables were $\mathrm{AL}$ or individual biomarkers. AL data were available for a subset of $186(90.3 \%)$ study participants. AL index values varied from 0 to 9 with a median of 1 and a mean of 1.70. Detailed statistics on individual biomarkers are available in the previously published manuscript [32].

In a univariate Poisson regression model, the estimated mean AL was 69\% (22\%; 135\%), $p=0.002$ greater among $T$. gondii seropositive individuals compared to seronegative controls (Model 4, Table 6). A multivariate model adjusting for age, race/ethnicity, education, and log-transformed BMI also produced a statistically significant association between $T$. gondii infection and $\mathrm{AL}$ (Model 5). The full predictive model for AL (Model 6) included, in addition to the above covariates, average distance-weighted vegetated land cover within $500 \mathrm{~m}$ radius of residence. The results show that greater vegetated land cover was a highly significant $(p<0.0001)$ negative predictor of AL indicating a beneficial effect of exposure (Table 6). The observed adjusted multiplicative effect on mean AL per IQR increase in vegetated land cover was $0.62(0.54 ; 0.72)$; in other words, the predicted mean AL was $38 \%(28 \%$; $46 \%)$ lower in individuals with greater vegetated land cover around their residences. The detrimental effect of $T$. gondii infection on AL was independent of the effect of green spaces: the mean AL was $61 \%(13 \% ; 130 \%)$ greater in seropositive individuals compared to seronegative controls in Model 6. 
Table 4 Results of multivariate regression analysis of predictors of T. gondii seropositivity; adjusted odds ratios (aOR) with 95\% confidence limits. Model 1 - logistic model for socio-demographic and behavioral predictors; Model 2 - logistic model with an interaction effect; Model 3 - generalized additive model including vegetated land cover

\begin{tabular}{|c|c|c|c|}
\hline Predictor & Model 1 & Model 2 & Model 3 \\
\hline Age (years) & $1.08(1.03,1.14)^{*}$ & $1.09(1.03,1.15)^{*}$ & $1.12(1.06,1.17)^{*}$ \\
\hline Current smoking & $5.74(1.29,25.6)^{*}$ & $5.16(1.11,23.9)^{*}$ & $7.68(1.78,33.1)^{*}$ \\
\hline Obesity & $2.76(0.67,11.4)$ & $4.67(1.01,21.6)^{*}$ & \\
\hline Eating undercooked pork & $5.68(0.81,39.9)$ & $4.89(0.71,33.7)$ & $10.1(1.52,67.7)^{*}$ \\
\hline Housing unit density, units/acre & $1.30(0.95,1.77)$ & & $1.30(0.92,1.83)$ \\
\hline Handling soil weekly & $5.34(1.37,20.7)^{*}$ & $0.35(0.03,4.19)$ & \\
\hline Currently owning a cat & $10.0(1.98,50.6)^{*}$ & & $12.1(2.86,51.5)^{*}$ \\
\hline Owning an outdoor cat & & $1.07(0.16,7.36)$ & \\
\hline Interaction effect of handling soil and owning outdoor cat & & $82.2(2.75,2454)^{*}$ & \\
\hline Two-dimensional spline function of geographic coordinates & & & $p=0.07$ \\
\hline Vegetated land cover within 500 m weighted, IQR increase & & & $3.67(1.48,9.08)^{*}$ \\
\hline
\end{tabular}

Additional stratified analysis in subsets of $T$. gondii seropositive (15 individuals with AL data) and seronegative $(N=171)$ study participants using a similar set of covariates demonstrated that exposure to vegetated land cover was statistically inversely associated with AL in both subsets. Multiplicative effect estimates per IQR increase in vegetated land cover were $0.26(0.14 ; 0.49)$, $p=0.004$ and $0.63(0.54 ; 0.73), p<0.0001$ in seropositive and seronegative individuals, respectively.

Further analysis focused on assessing effects of latent T. gondii infections on dichotomized biomarkers. Regression models included age, sex, race/ethnicity, education, smoking status, and log-transformed BMI as covariates. Adjusted odds ratios of having potentially unhealthy biomarker values either below the 10th percentile or above the 90th percentile of sample distribution are presented in Fig. 1. Serum MPO was the only biomarker significantly associated with $T$. gondii infection with 9.85 (2.36; 41.0), $p=0.0017$ aOR of biomarker value above the 90th percentile in seropositive individuals compared to seronegative controls. The observed association with MPO remained statistically significant after applying a Bonferroni correction for conducting exploratory analysis of 17 biomarkers, which reduced the cut-off for statistical significance to $\alpha^{*}=0.05 / 17=$
0.003. The effect estimates for 12 of 17 (71\%) biomarkers were greater than one (Fig. 1) suggesting increased odds of having potentially unhealthy biomarker values in $T$. gondii seropositive individuals. The predominance of positive associations with dichotomized biomarkers explains the highly statistically significant effect of $T$. gondii on AL measures comprised of these biomarkers.

Analysis of associations between $T$. gondii seropositivity and continuous log-transformed biomarkers involved a similar set of covariates. The results (Fig. 2) demonstrated that $T$. gondii seropositivity was associated with $25 \%$ (9\%; 44\%) higher adjusted median concentration of serum MPO ( $p=0.001), 50 \%(1 \% ; 123 \%)$ higher level of IL-6 $(p=0.04)$ and $7 \%(0.3 \% ; 13 \%)$ higher level of VCAM-1 $(p=0.04)$. Only the association with MPO remained statistically significant after applying a Bonferroni correction for multiple testing.

\section{Discussion}

In this study of urban and suburban adult residents of central North Carolina, greater residential vegetated land cover was significantly associated with $T$. gondii seropositivity. In previous studies, exposures to urban green spaces and other natural living environments have been linked to reduced morbidity and mortality; however,

Table 5 Comparison of associations between vegetated land cover measure and $T$. gondii seropositivity using generalized additive models (covariates as in Model 3 in Table 4)

\begin{tabular}{llll}
\hline Vegetated land cover measure & IQR, \% & Model deviance & $\begin{array}{l}\text { aOR of T. gondii seropositivity with } \\
95 \% \text { confidence limits }\end{array}$ \\
\hline $50 \mathrm{~m}$ average & 25.9 & 68.94 & $2.39(1.09,5.24)^{*}$ \\
$150 \mathrm{~m}$ average & 21.7 & 68.37 & $2.85(1.20,6.76)^{*}$ \\
$500 \mathrm{~m}$ average & 15.0 & 67.79 & $3.01(1.29,7.00)^{*}$ \\
$500 \mathrm{~m}$ weighted average & 17.4 & 67.13 & $3.67(1.48,9.08)^{*}$ \\
\hline
\end{tabular}


Table 6 Results of regression analysis of predictors of AL, adjusted multiplicative effects on AL with 95\% confidence limits

\begin{tabular}{|c|c|c|c|}
\hline Predictor variable & Model 4 & Model 5 & Model 6 \\
\hline Age, year & & $1.01(1.001,1.02)^{*}$ & $1.01(1.004,1.02)^{*}$ \\
\hline Non-Hispanic White race/ethnicity & & $1.20(0.94,1.54)$ & $1.37(1.06,1.78)^{*}$ \\
\hline Education (bachelor's degree or higher) & & $0.83(0.65,1.06)$ & $0.73(0.57,0.94)^{*}$ \\
\hline $\log _{10}(B M I)$ & & $12.7(4.15,39.1)^{*}$ & $10.8(3.57,32.9)^{*}$ \\
\hline Vegetated land cover, per IQR increase & & & $0.62(0.54,0.72)^{*}$ \\
\hline $\begin{array}{l}\text { Two-dimensional spline function of } \\
\text { geographic coordinates }\end{array}$ & & & $p=0.04$ \\
\hline T. gondii seropositivity & $1.69(1.22,2.35)^{*}$ & $1.43(1.01,2.03)^{*}$ & $1.63(1.15,2.33)^{*}$ \\
\hline
\end{tabular}

evidence of potential detrimental effects of green spaces including zoonotic infections remained rather sparse [37]. To our knowledge, this is the first study linking residential greenness to $T$. gondii infections.

The present study also found that handling soil with bare hands was a risk factor for $T$. gondii seropositivity and produced some evidence suggesting that individuals living in a greener residential environment were more likely to acquire infection through contacts with local soil contaminated with environmental oocysts of this parasite. The association between contacts with soil and T. gondii infections is corroborated by previous research $[15,16,18]$. A study in France also demonstrated that urban and rural residents acquired T. gondii infections through different pathways: parasites isolated from urban residents lacked geographic genetic structure, suggesting foodborne infections via products transported

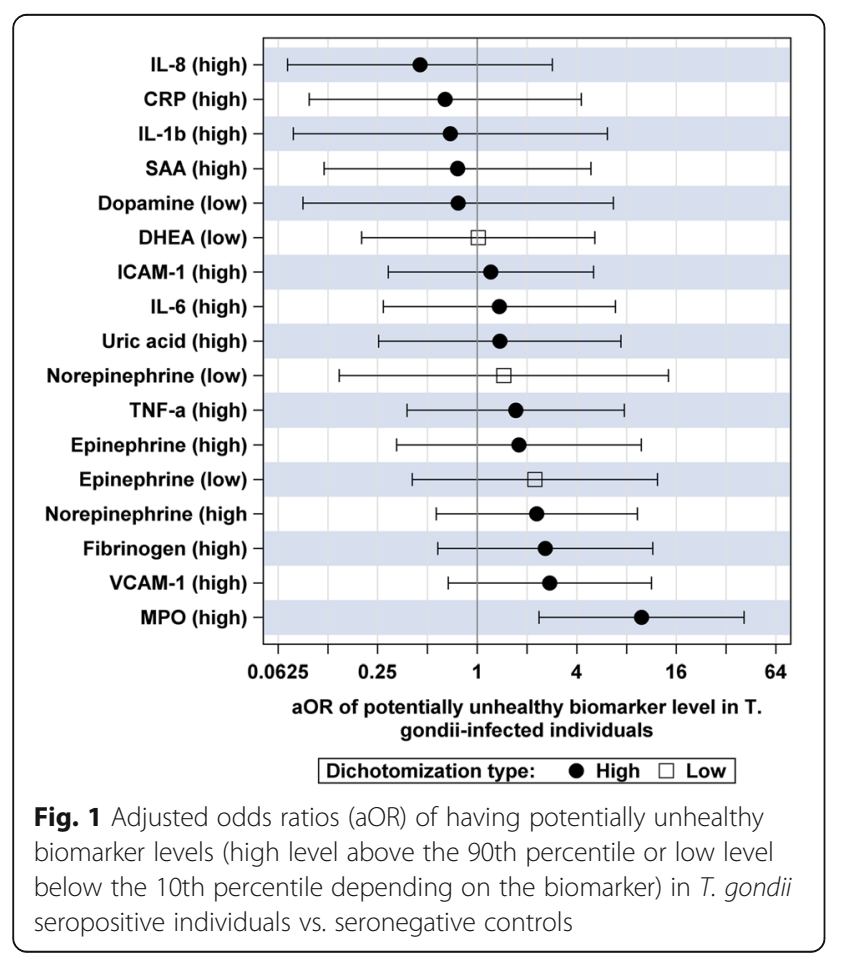

over long distances; in contrast, parasites isolated from rural residents exhibited a spatial genetic structure, suggesting a greater importance of local sources of infection [38].

The observed $8.3 \%$ seroprevalence of $T$. gondii in this study involving adults was lower than the $13.2 \%$ national estimate of unadjusted seroprevalence in children $(>5$ years of age) and adults in the 2009-2010 NHANES study [8]. The present study was conducted in an urban area with a relatively high educational and socioeconomic status compared to the general US population, which may explain the relatively low observed seroprevalence rate. Local conditions affecting the spread of $T$. gondii might also differ from those in other US regions. Recruitment methods for this study attempted to oversample cat or dog owners. Cat ownership was a risk factor for T. gondii seropositivity; this finding was consistent with results of previously conducted studies [10, 11, 14]. Having a dog was a negative predictor of cat ownership; it also tended to be inversely

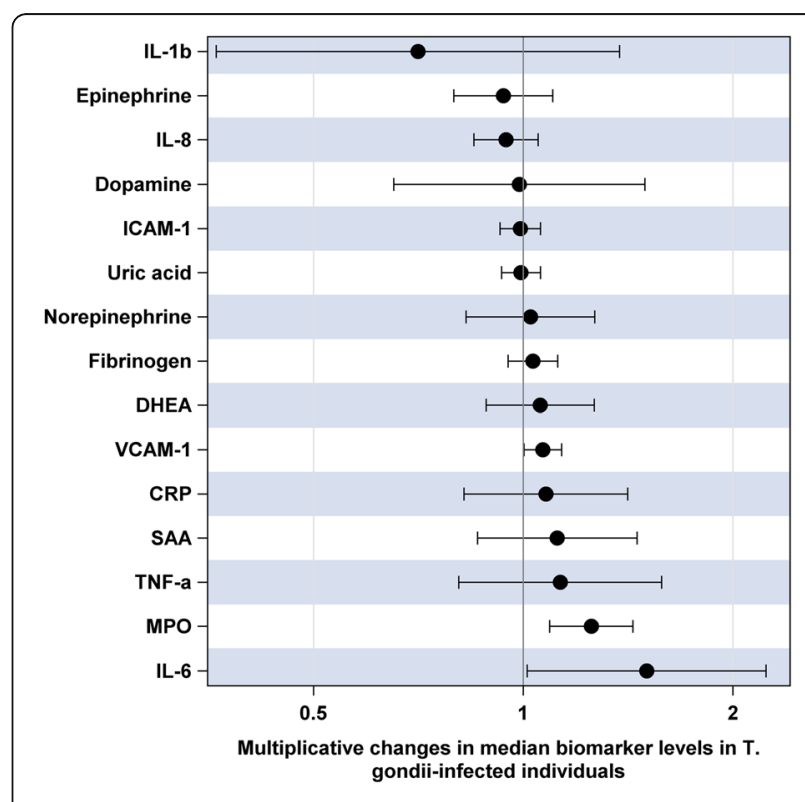

Fig. 2 Adjusted multiplicative changes in median biomarker levels in T. gondii seropositive individuals compared to seronegative controls 
associated with $T$. gondii seropositivity, although the effect was not statistically significant. This finding is consistent with previous research which demonstrated a significant protective effect of dog ownership on $T$. gondii infections [39]. Thus, the effects of oversampling cat and dog owners on T. gondii seropositivity in the study population could cancel out each other. Although the seroprevalence estimate from this study is not generalizable due to non-random sampling, findings about risk factors for $T$. gondii infections are likely to reflect transmission pathways of this parasite in central North Carolina.

Furthermore, the present study showed that latent $T$. gondii infections were associated with a detrimental systemic effect reflected in elevated $\mathrm{AL}$, a composite measure of physiologic dysregulation based on multiple biomarkers. Our previous study in the same group of adult residents of North Carolina demonstrated that greener residential environment was associated with reduced $\mathrm{AL}$ [32]. Thus, T. gondii infections acquired through more frequent contacts with contaminated soils in greener neighborhoods where cats were allowed to roam outdoors could partially offset health benefits of contacts with the natural living environment. This finding shows the importance of minimizing risks of zoonotic infections in green areas.

A limitation of this cross-sectional observational study is that it could only demonstrate statistical associations; it was not designed to establish a cause-effect relationship. While one of the hypotheses of this study was that latent $T$. gondii infections cause chronic inflammation resulting in greater physiological dysregulation and elevated AL measures, there may be alternative explanations for the observed effect. It is possible that individuals with higher AL were more susceptible to $T$. gondii infections (reverse causation). It is also possible that the observed association was due to confounding by unknown behavioral factors that affect the risk of T. gondii infection as well as AL.

The beneficial effect of residential vegetation on $\mathrm{AL}$ demonstrated in our previous study in the same population [32] remained highly significant after adjusting for $T$. gondii serostatus in the present study. Further stratified analysis showed that the beneficial effects of vegetated land cover were pronounced in both seropositive and seronegative individuals. In seropositive individuals, the detrimental effect of $T$. gondii and the beneficial effect of an IQR increase in residential greenery were of similar magnitudes. However, due to the small sample size (only 15 seropositive individuals with $\mathrm{AL}$ data), these effect estimates should be interpreted with caution.

Analysis of associations with individual biomarkers, which comprised the AL index, showed that $T$. gondii infection was linked with a significantly increased aOR of having serum MPO above the 90th percentile and, in analysis of continuous biomarker data, with a higher median concentration of MPO. These findings are logical as MPO is an enzyme involved in immune response to pathogens. Elevated levels of MPO have been linked with inflammation and with cardiovascular diseases [40, 41]. T. gondii seropositivity was also linked with elevated levels of VCAM-1 and IL-6 ( $p<0.05$ for each association), although these effects did not remain significant after applying Bonferroni correction for multiple comparisons. Previous research demonstrated associations between $T$. gondii infection and an elevated serum level of VCAM-1 and some pro-inflammatory cytokines [31] corroborating the results of this study. Experimental research also demonstrated that pro-inflammatory cytokines including IL-6 and TNF- $\alpha$ play critical roles in inhibiting the replication of this parasite in humans [42]. Previous studies have also shown associations between $T$. gondii and elevated serum ICAM-1 [43], and reduced serum DHEA (both associations suggesting detrimental health effects) [44]. Those findings were not replicated in the present study possibly due to the small sample size or particular characteristics of the study population.

\section{Conclusions}

The results of this study suggest that handling soil with bare hands, having an outdoor cat and having more greenery around residence were risk factors for $T$. gondii infection in residents of central North Carolina. In turn, T. gondii IgG seropositivity was associated with an increased AL suggesting that latent infections have subtle detrimental effects leading to physiological dysregulation and potentially enhancing risk of overt diseases. In contrast, having more greenery around residence was associated with reduced AL in both seronegative and seropositive individuals. The zoonotic $T$. gondii infections may partially offset health benefits of green spaces in a subset of the population.

\section{Abbreviations}

AL: Allostatic load; aOR: adjusted odds ratio; CRP: C-reactive protein;

DHEA: Dehydroepiandrosterone; ICAM-1: Intercellular adhesion molecule 1; Ig: Immunoglobulin; IL: Interleukin; IQR: Interquartile range;

MPO: Myeloperoxidase; SAA: Serum amyloid A; TNF-a: Tumor necrosis factor a; US EPA: United States Environmental Protection Agency; VCAM-1: Vascular cell adhesion molecule 1

\footnotetext{
Acknowledgements

The authors are grateful to Dr. Laura Jackson and the EnviroAtlas land cover classification team at US EPA for developing the land cover dataset and providing consultations on its use, and to Dr. Eric Villegas (US EPA) for helpful comments on a draft manuscript. This study was an intramural research project at US EPA. Jennifer N. Styles was funded by the US EPA-UNC-CH Cooperative Training Agreement CR-83591401-0, with the Department of Environmental Sciences and Engineering, University of North Carolina at Chapel Hill.
}

Funding

This study was conducted by US EPA. No external funding was used. 


\section{Availability of data and materials}

The datasets used and/or analyzed during the current study are available from the corresponding author on reasonable request.

\section{Disclaimer}

The research described in this article has been reviewed by the National Health and Environmental Effects Research Laboratory, U.S. Environmental Protection Agency, and approved for publication. Approval does not signify that the contents necessarily reflect the views and policies of the Agency, nor does the mention of trade names of commercial products constitute endorsement or recommendation for use.

\section{Authors' contributions}

RC, TJW, AIE and SMG contributed to design of the study. RC, ES, EH, and TJW organized field data collection. RC, JS, SMG, and AIE conducted laboratory analysis of serum samples. EK analyzed GIS data. AIE conducted statistical data analysis and drafted the manuscript with intellectual contributions from RC, SMG, and TJW. All authors read and approved the final manuscript.

\section{Ethics approval and consent to participate}

Research reported in this study has been performed with the approval of the ethics committee of the University of North Carolina at Chapel Hill. Human research was carried out in compliance with the Helsinki Declaration. Written consent was obtained from all participants prior to data collection.

\section{Consent for publication}

Not applicable.

\section{Competing interests}

The authors declare that they have no competing interests.

\section{Publisher's Note}

Springer Nature remains neutral with regard to jurisdictional claims in published maps and institutional affiliations.

\section{Author details}

${ }^{1}$ United States Environmental Protection Agency, National Health and Environmental Effects Research Laboratory, MD 58-C, 109. T.W. Alexander Drive, Research Triangle Park, NC 27711, USA. ${ }^{2}$ United States Environmental Protection Agency, National Exposure Research Laboratory, Cincinnati, $\mathrm{OH}$, USA. ${ }^{3}$ Gillings School of Global Public Health, Environmental Sciences and Engineering Department, University of North Carolina at Chapel Hill, Chapel Hill, NC, USA. ${ }^{4}$ ORAU Student Services Contractor to US EPA, Chapel Hill, NC, USA.

\section{Received: 28 February 2018 Accepted: 17 August 2018}

\section{Published online: 23 August 2018}

\section{References}

1. Lelu M, Villena I, Darde ML, Aubert D, Geers R, Dupuis E, et al. Quantitative estimation of the viability of Toxoplasma gondii oocysts in soil. Appl Environ Microbiol. 2012;78(15):5127-32.

2. Dabritz HA, Miller MA, Atwill ER, Gardner IA, Leutenegger CM, Melli AC, et al. Detection of Toxoplasma gondii-like oocysts in cat feces and estimates of the environmental oocyst burden. J Am Vet Med Assoc. 2007;231(11):1676-84.

3. Jones J, Lopez A, Wilson M. Congenital toxoplasmosis. Am Fam Physician. 2003;67(10):2131-8.

4. Robert-Gangneux F, Darde ML. Epidemiology of and diagnostic strategies for toxoplasmosis. Clin Microbiol Rev. 2012;25(2):264-96.

5. Jones JL, Parise ME, Fiore AE. Neglected parasitic infections in the United States: toxoplasmosis. Am J Trop Med Hyg. 2014;90(5):794-9.

6. Halonen SK, Weiss LM. Toxoplasmosis. Handbook Clin Neurol. 2013;114:125-45.

7. Jones JL, Kruszon-Moran D, Wilson M, McQuillan G, Navin T, McAuley JB. Toxoplasma gondii infection in the United States: seroprevalence and risk factors. Am J Epidemiol. 2001;154(4):357-65.

8. Jones $J$, Kruszon-Moran D, Rivera HN, Price C, Wilkins PP. Toxoplasma gondii seroprevalence in the United States 2009-2010 and comparison with the past two decades. Am J Trop Med Hyg. 2014;90(6):1135-9.

9. Krueger WS, Hilborn ED, Converse RR, Wade TJ. Drinking water source and human Toxoplasma gondii infection in the United States: a cross-sectional analysis of NHANES data. BMC Public Health. 2014;14:711.
10. Jones JL, Dargelas V, Roberts J, Press C, Remington JS, Montoya JG. Risk factors for Toxoplasma gondii infection in the United States. Clin Infect Dis. 2009;49(6):878-84

11. Kolbekova P, Kourbatova E, Novotna M, Kodym P, Flegr J. New and old riskfactors for Toxoplasma gondii infection: prospective cross-sectional study among military personnel in the Czech Republic. Clin Microbiol Infect. 2007; 13(10):1012-7.

12. Hill D, Coss C, Dubey JP, Wroblewski K, Sautter M, Hosten T, et al. Identification of a sporozoite-specific antigen from Toxoplasma gondii. J Parasitol. 2011;97(2):328-37.

13. Boyer K, Hill D, Mui E, Wroblewski K, Karrison T, Dubey JP, et al. Unrecognized ingestion of Toxoplasma gondii oocysts leads to congenital toxoplasmosis and causes epidemics in North America. Clin Infect Dis. 2011;53(11):1081-9.

14. Hofhuis A, van Pelt W, van Duynhoven YT, Nijhuis CD, Mollema L, van der Klis FR, et al. Decreased prevalence and age-specific risk factors for Toxoplasma gondii lgG antibodies in The Netherlands between 1995/1996 and 2006/2007. Epidemiol Infect. 2011;139(4):530-8.

15. Cook AJ, Gilbert RE, Buffolano W, Zufferey J, Petersen E, Jenum PA, et al. Sources of toxoplasma infection in pregnant women: European multicentre case-control study. European Research Network on Congenital Toxoplasmosis. BMJ. 2000;321(7254):142-7.

16. Nash JQ, Chissel S, Jones J, Warburton F, Verlander NQ. Risk factors for toxoplasmosis in pregnant women in Kent, United Kingdom. Epidemiol Infect. 2005;133(3):475-83.

17. Taylor MR, Lennon B, Holland CV, Cafferkey M. Community study of toxoplasma antibodies in urban and rural schoolchildren aged 4 to 18 years. Arch Dis Child. 1997;77(5):406-9.

18. Dabritz HA, Conrad PA. Cats and Toxoplasma: implications for public health. Zoonoses Public Health. 2010;57(1):34-52.

19. Bowie WR, King AS, Werker DH, Isaac-Renton JL, Bell A, Eng SB, et al. Outbreak of toxoplasmosis associated with municipal drinking water. The BC Toxoplasma Investigation Team. Lancet. 1997;350(9072):173-7.

20. de Moura L, Bahia-Oliveira LM, Wada MY, Jones JL, Tuboi SH, Carmo EH, et al. Waterborne toxoplasmosis, Brazil, from field to gene. Emerg Infect Dis. 2006;12(2):326-9.

21. Weiss LM, Dubey JP. Toxoplasmosis: A history of clinical observations. Int J Parasitol. 2009;39(8):895-901.

22. Fekadu A, Shibre T, Cleare AJ. Toxoplasmosis as a cause for behaviour disorders--overview of evidence and mechanisms. Folia Parasitol (Praha). 2010;57(2):105-13.

23. Hampton MM. Congenital Toxoplasmosis: A Review. Neonatal Netw. 2015; 34(5):274-8.

24. Vyas A, Kim SK, Giacomini N, Boothroyd JC, Sapolsky RM. Behavioral changes induced by Toxoplasma infection of rodents are highly specific to aversion of cat odors. Proc Natl Acad Sci U S A. 2007;104(15):6442-7.

25. House PK, Vyas A, Sapolsky R. Predator cat odors activate sexual arousal pathways in brains of Toxoplasma gondii infected rats. PloS One. 2011;6(8): e23277.

26. Poirotte C, Kappeler PM, Ngoubangoye B, Bourgeois S, Moussodji M, Charpentier MJ. Morbid attraction to leopard urine in Toxoplasma-infected chimpanzees. Curr Biol. 2016;26(3):R98-9.

27. Ling VJ, Lester D, Mortensen PB, Langenberg PW, Postolache $\Pi$. Toxoplasma gondii seropositivity and suicide rates in women. J Nerv Ment Dis. 2011;199(7):440-4.

28. Flegr J, Klose J, Novotna M, Berenreitterova M, Havlicek J. Increased incidence of traffic accidents in Toxoplasma-infected military drivers and protective effect RhD molecule revealed by a large-scale prospective cohort study. BMC Infect Dis. 2009;9:72.

29. Majidiani H, Dalvand S, Daryani A, Galvan-Ramirez ML. Foroutan-Rad M. Is chronic toxoplasmosis a risk factor for diabetes mellitus? A systematic review and meta-analysis of case-control studies. Braz J Infect Dis. 2016; 20(6):605-9.

30. Tedford E, McConkey G. Neurophysiological Changes Induced by Chronic Toxoplasma gondii Infection. Pathogens. 2017;6(2)

31. Tomasik J, Schultz TL, Kluge W, Yolken RH, Bahn S, Carruthers VB. Shared Immune and Repair Markers During Experimental Toxoplasma Chronic Brain Infection and Schizophrenia. Schizophr Bull. 2016;42(2):386-95.

32. Egorov Al, Griffin SM, Converse RR, Styles JN, Sams EA, Wilson A, et al. Vegetated land cover near residence is associated with reduced allostatic load and improved biomarkers of neuroendocrine, metabolic and immune functions. Environ Res. 2017;158:508-21. 
33. Juster RP, BS ME, Lupien SJ. Allostatic load biomarkers of chronic stress and impact on health and cognition. Neurosci Biobehav Rev. 2010;35(1):2-16.

34. Dormann C, McPherson J, Araújo M, Bivand R, Bolliger J, Carl G, et al. Methods to account for spatial autocorrelation in the analysis of species distributional data: a review. Ecography. 2007;30(5):609-28.

35. Clements MS, Armstrong BK, Moolgavkar SH. Lung cancer rate predictions using generalized additive models. Biostatistics. 2005;6(4):576-89.

36. Webster T, Vieira V, Weinberg J, Aschengrau A. Method for mapping population-based case-control studies: an application using generalized additive models. Int J Health Geogr. 2006;5:26.

37. WHO. Urban Green Spaces and Health-A Review of Evidence. Geneva: WHO; 2016. Accessed at: http://www.euro.who.int/_data/assets/pdf_file/ 0005/321971/Urban-green-spaces-and-health-review-evidence.pdf

38. Ajzenberg D, Collinet F, Aubert D, Villena I, Darde ML, French ToxoBs network $\mathrm{g}$, et al. The rural-urban effect on spatial genetic structure of type II Toxoplasma gondii strains involved in human congenital toxoplasmosis, France, 2002-2009. Infect Genet Evol. 2015;36:511-6.

39. Yang N, Wang D, Xing M, Li C, Li J, Wu A, et al. Seroepidemiology and Risk Factors of Toxoplasma gondii Infection among the Newly Enrolled Undergraduates and Postgraduate Students in China. Front Microbiol. 2017:8:2092.

40. Loria V, Dato I, Graziani F, Biasucci LM. Myeloperoxidase: a new biomarker of inflammation in ischemic heart disease and acute coronary syndromes. Mediators Inflamm. 2008;2008:135625.

41. Zakynthinos E, Pappa N. Inflammatory biomarkers in coronary artery disease. J Cardiol. 2009;53(3):317-33.

42. Chao CC, Gekker G, Hu S, Peterson PK. Human microglial cell defense against Toxoplasma gondii. The role of cytokines. J Immunol. 1994;152(3): 1246-52.

43. El-Sayed NM, Ismail KA, Ahmed SA-E-G, Ezz-El-Din HM, HME-E-D A. Possible association between Toxoplasma gondii infection and schizophrenia: Egyptian study. Infect Dis Clin Pract. 2012;20(6):394-9.

44. Flegr J, Priplatova L, Hampl R, Bicikovia M, Ripova D, Mohr P. Difference of neuro- and immunomodulatory steroids and selected hormone and lipid concentrations between Toxoplasma-free and Toxoplasma-infected but not CMV-free and CMV-infected schizophrenia patients. Neuro Endocrinol Lett. 2014;35(1):20-7.

Ready to submit your research? Choose BMC and benefit from:

- fast, convenient online submission

- thorough peer review by experienced researchers in your field

- rapid publication on acceptance

- support for research data, including large and complex data types

- gold Open Access which fosters wider collaboration and increased citations

- maximum visibility for your research: over $100 \mathrm{M}$ website views per year

At $\mathrm{BMC}$, research is always in progress.

Learn more biomedcentral.com/submissions 